\title{
Role of Prevalent Weeds and Cultivated Crops in the Epidemiology of Maize Lethal Necrosis Disease in Major Maize Growing Agroecological Zones of Uganda
}

\author{
Barnabas Mudde ${ }^{1,2^{*}}$, Dora C. Kilalo², Florence M'mogi Olubayo ${ }^{2}$, Godfrey Asea ${ }^{3}$, \\ Andrew Kiggundu ${ }^{4}$, Daniel Bomet Kwemoi ${ }^{3}$ and Douglas Watuku Miano ${ }^{2}$ \\ ${ }^{1}$ National Agricultural Research Organisation, Bulindi Zonal Agricultural Research and Development \\ Institute, P.O.Box 101, Hoima, Uganda. \\ ${ }^{2}$ Department of Plant Science and Crop Protection, Faculty of Agriculture, College of Agriculture and \\ Veterinary Sciences, University of Nairobi, P.O.Box 29053, Nairobi, Kenya. \\ ${ }^{3}$ National Crops Resources Research Institute, Namulonge, P.O.Box 7084, Kampala, Uganda. \\ ${ }^{4}$ National Agricultural Research Laboratories, Kawanda, P.O.Box 7065, Kampala, Uganda.
}

\section{Authors' contributions}

This work was carried out in collaboration among all authors. Author BM designed the study, performed the statistical analysis, managed the literature searches, interpreted the data, wrote the protocol and the first draft of the manuscript. Authors DCK, FMO, GA, AK, DBK and DWM contributed

to data analysis, data interpretation and critical revision of the article. All authors read and approved the final manuscript.

Article Information

DOI: 10.9734/ARRB/2019/v32i330084

Editor(s):

(1) Dr. Amit Kesarwani, Assistant Professor, Department of Agronomy, College of Agriculture, G.B. Pant University of Agriculture \& Technology, India.

(2) Dr. George Perry, Dean and Professor of Biology, University of Texas at San Antonio, USA. Reviewers:

(1) Marcio Martinello Sanches, Embrapa Recursos Geneticos e Biotecnologia, Brasil. (2) Marysol Alvear, Universidad De La Frontera, Chile. (3) Clint Magill, Texas A\&M University, USA. Complete Peer review History: http://www.sdiarticle3.com/review-history/49407

Review Article

Received 15 April 2019

Accepted 21 June 2019

Published 08 July 2019

\section{ABSTRACT}

In Uganda, the severe Maize lethal necrosis (MLN) disease, which threatens subsistence maize production is caused by co-infection of maize plants with Maize chlorotic mottle virus (MCMV) and Sugarcane mosaic virus (SCMV). However, there is no information about natural hosts of MLN causing viruses and their role in epidemiology of MLN in Uganda. The aim of this study was to 
determine existence of natural alternative weed and cultivated crop hosts of MLN causing viruses. Three seasonal surveys between 2014 and 2015 were carried out in five major maize growing agroecological zones of Uganda. Weeds and cultivated crops growing in proximity to maize were observed for virus symptoms and tested for MLN causing viruses using Double Antibody Sandwich Enzyme-Linked Immunosorbent Assay and Reverse Transcriptase Polymerase Chain Reaction. Data was collected on frequency of occurrence of weeds and cultivated crop species and MLN virus disease incidence. Digitaria abyssinica, Bidens pilosa and Commelina benghalensis were the most common weed species while Phaseolus vulgaris, Manihot esculenta, Arachis hypogaea), Musa sp, Glycine max and Ipomoea batatas were most common cultivated crops. Pennisetum purpureum, Digitaria abyssinica, Cyperus rotundus, Amaranthus spinosus, Commelina benghalensis and Eleusine indica weeds species are natural hosts of Maize chlorotic mottle virus. Among the cultivated crops, Phaseolus vulgaris, Manihot esculenta and Sesamum indicum are natural hosts of MCMV. Only Sorghum (Sorghum bicolor) and sweet potato (Ipomoea batatas) tested positive for SCMV. MCMV incidence in weeds ranged from $2 \%$ to $63 \% \%$ and $2 \%$ to $29 \%$ in cultivated crops. MLN causing viruses were prevalent in weeds and cultivated crops located in known hotspots for MLN in Uganda. The study has revealed that alternative hosts of MLN-causing viruses are present in major maize growing agroecological zones of Uganda and act as sources of inoculum to sustain MLN epidemics.

Keywords: Alternative weed hosts; epidemiology; maize lethal necrosis; Uganda.

\section{INTRODUCTION}

Maize lethal necrosis (MLN) disease has been reported in different countries of East and Central Africa including Rwanda [1], Tanzania [2], Kenya [3], Uganda [4,5] and Democratic Republic of Congo [6] and is now considered to be the most widespread and serious virus disease on maize in sub-Saharan Africa. MLN is not indigenous to the African continent. In Uganda, it is not yet widespread and abundant. The disease is caused when maize plants are co-infected with Maize chlorotic mottle virus and other cereal viruses in the potyvirus group such as Maize dwarf mosaic virus (MDMV) and Sugarcane mosaic virus (SCMV). However apart from maize, there is scanty information about its natural alternative hosts and ecology in Uganda. Maize was the only naturally occurring host of MCMV known [7], until recently when the virus was detected in sugarcane [8] and finger millet [9]. These new findings point to the possibility of other new natural hosts of MLN viruses that could be present in Uganda. In addition, MCMV has been reported to have a broad experimental host range including no less than 19 grass species making it difficult to manage the virus [10]. The wide host range has implications on the epidemiology of virus diseases and should be considered in development of an integrated disease management strategy.

Several studies on the significance of weeds as reservoirs of MLN causing viruses notably MCMV $[8,11,7,10]$ and MDMV and SCMV
$[12,13,14]$ have been conducted in maize production agro-ecologies in temperate regions of North America. Little or no related studies have been conducted in tropical regions $[9,15,16]$. Differences in agroecosystems and agro-ecologies result in differences in the biology and occurrence of different weed species, which may cause variation in their relation to MLN causing viruses and their vectors. These alternative weed hosts growing in association with maize agroecosystems in countries where first studies on MLN were conducted may have acted as alternative hosts for vectors or as reservoirs of the MLN causing viruses or both. However, it is not known whether similar grass weed species that are hosts of MLN are present in Uganda and if they could have had a role in enhancing the spread of MLN. The alternative host status of related plant species and common intercrops grown with maize in Uganda for the Ugandan strain(s) of MLN causing viruses and their role in the spread of MLN in Uganda is unknown. Some maize viruses have been known to have different reactions on the same alternative host due to variation in the strains. There have been no studies conducted on MLN causing viruses and their natural or artificial hosts in Uganda. Some non-chemical methods of managing MLN such as crop rotation and fallowing focus on the removal of the maize host from the field for a defined period. It is not known whether crop rotation is a feasible MLN disease control measure. The success of such cultural methods depends on the duration of survival of MLN causing viruses without a maize host or 
alternate hosts. In most cases no attention is given to weeds which could be alternative hosts and sources of inoculum for MLN viruses. In order to develop an MLN management strategy that is effective, information should be generated through studies conducted to establish the host range of Ugandan strain(s) of MLN causing viruses. The aim of this study was to establish the weeds and cultivated crop species occurring in maize agroecological zones of Uganda and to determine the existence of natural alternative weed and cultivated crop hosts of MLN causing viruses. It was hypothesized that potential alternative hosts of Maize Lethal Necrosiscausing viruses are present in major maize growing agroecological zones of Uganda and act as natural sources of inoculum to maize fields.

\section{MATERIALS AND METHODS}

\subsection{Description of the Study Area}

The study was conducted over three seasons from 2014A, 2014B and 2015A and covered 14 major maize growing districts from five agroecological zones (AEZ) of Uganda (Fig. 1). Where " $A$ " is the first rainy season (March-July) and " $B$ " is the second rainy season (SeptemberDecember). The study agroecological zones were classified according to the National Agricultural Research Organization [17] based on distinct vegetation type, elevation and climatic conditions. The Eastern Agro-ecological Zone (EAZ) covered the districts of Tororo and Bukedea and is characterized by an annual average rainfall of $1197 \mathrm{~mm}$ with temperature ranges from $15-32.5^{\circ} \mathrm{C}$. The Lake Albert Crescent Agro-ecological Zone (LACZ) covered the districts of Hoima, Masindi, Kibaale and Kiryandongo and is characterized by an annual average rainfall of $1259 \mathrm{~mm}$ with temperature ranging from $17.5-32.5^{\circ} \mathrm{C}$. The South Eastern Agro-ecological Zone (SEAZ) covered the districts of Iganga, Busia and Bugiri with average annual rainfall ranging from $1215-1328 \mathrm{~mm}$ and temperature ranging from 15 to $32.5^{\circ} \mathrm{C}$. The Eastern Highlands Agro-ecological Zone (EHAZ) covered the districts of Mbale, Bulambuli,

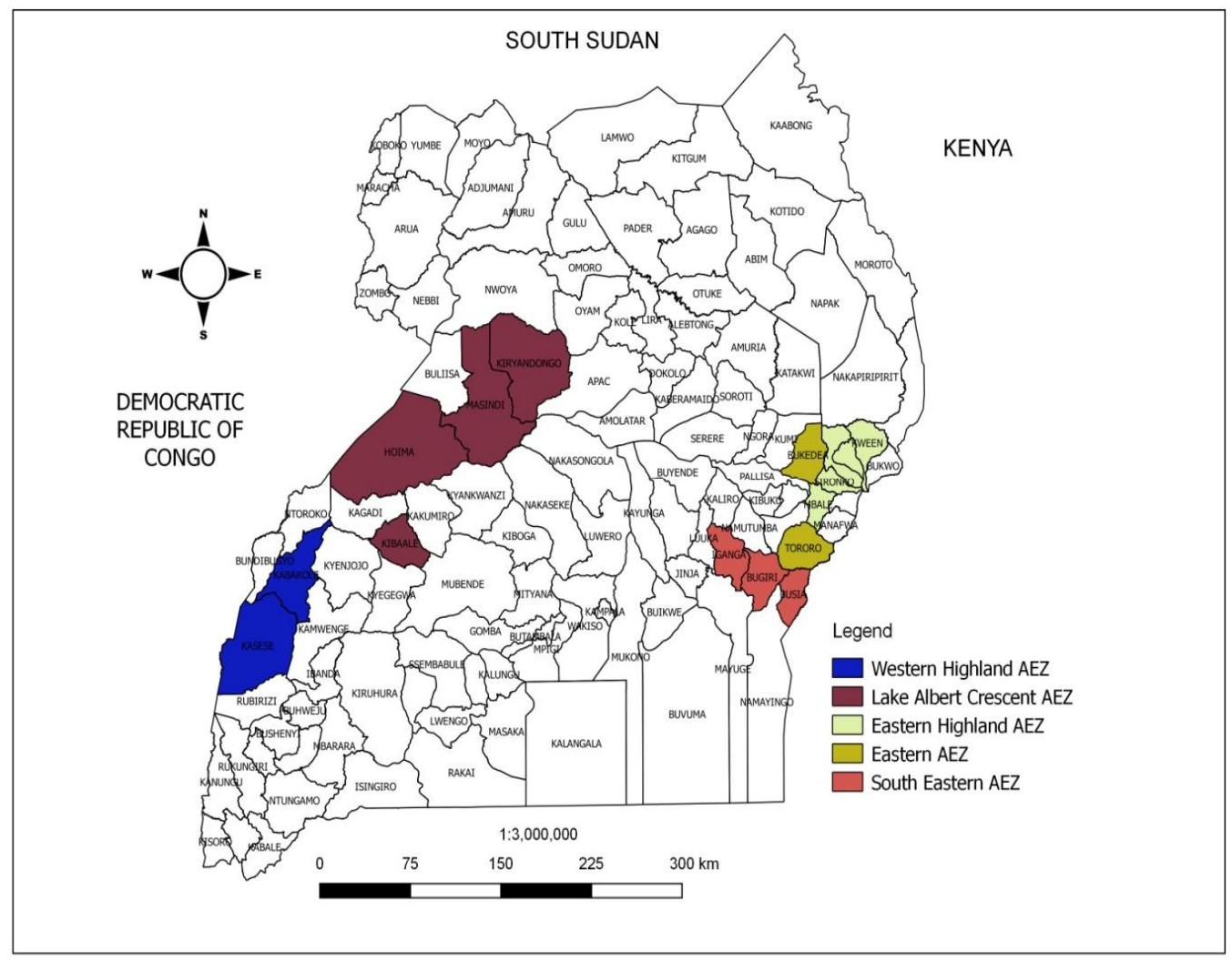

Fig. 1. The location of the five major maize growing agroecological zones in Uganda surveyed for alternative hosts of MLN viruses during three seasons. Where AEZ= Agroecological Zone 
Sironko, Kween and Kapchorwa with average annual rainfall more than $1,400 \mathrm{~mm}$ and temperature ranging from 7.5 to $27.5^{\circ} \mathrm{C}$. The Western Highlands Agro-ecological Zone (WHAZ) covered the districts of Kasese and Kabarole with average annual rainfall of $1,270 \mathrm{~mm}$ and temperature ranging from 15 to $30^{\circ} \mathrm{C}$.

\subsection{Field Surveys and Sampling}

The five major maize growing agroecological zones of Uganda (namely, the Eastern, Eastern Highland, South Eastern, Western Highland and Lake Albert Crescent Agroecological zones) were surveyed for alternative host plants of MLN. From each agroecological zone, at least two districts were chosen purposively based on maize production data and surveyed for plants that were displaying virus-like symptoms or were apparently healthy looking. The surveys were carried out during the first season of 2014 , second season of 2014 and first season of 2015 from 16 major maize growing districts. Fields were selected at regular intervals along major and feeder roads traversing the sampling area. Average distance between sampled fields was about $5 \mathrm{~km}$. Weeds and cultivated crops grown as intercrops or near maize were collected from near maize fields on either side of the road while alternating after every $5 \mathrm{~km}$. In all cases, the site of collection was a maize field in which the plants were growing naturally as weeds. Sampling activities commenced in the early morning hours just before sunrise (between 0630 and $0700 \mathrm{~h}$ ) to minimize the impact of wilting. Cultivated crop species and weeds growing within the vicinity of the maize crop and one meter from the maize plant were collected together with their rooting system and crown. At each stop the 3 plants from each weed species and 3 plants from each intercrop species grown with maize in a 1-meter square area was sampled using a quadrat. The samples collected were put in separate bags to avoid cross contamination. A total of 10 sampling sites were located 5 to $10 \mathrm{~km}$ from each other.

\subsection{Plant Materials and Taxonomic Identification}

Plants were initially identified during the field surveys only by close examination for distinguishing features of common weed and crop families. The plants were later identified to species level according to the taxonomic keys using reference herbarium collections available at the Department of Botany Herbarium, Makerere University using reference identification keys from [18].

\subsection{Detection of Viruses in Weeds and Cultivated Crops from Five Major Maize Growing Agroecological Zones of Uganda}

Serological detection of Maize lethal necrosis causing viruses in weeds and cultivated crops: Between 5 and 10 leaves (preferably those with virus-like symptoms) were sampled from taxonomically identified plants. Leaves were used for serological testing of MLN-causing viruses notably MCMV and SCMV based on previous findings that identified them as the key MLN causing viruses present in Uganda [19]. Double antibody sandwich enzyme-linked immunosorbent assay (DAS-ELISA) was used to test for the presence of SCMV and MCMV in weed and crop leaf samples collected during the survey [20]. The antisera were purchased from Agdia Inc. (Elkhart, IN, USA). The polyclonal antibodies used included antiSugarcane mosaic virus and anti- Maize chlorotic mottle virus. In the test all the buffers were prepared according to the manufacturer's specifications from Agdia Inc. (Elkhart, IN, USA).

Plant leaf samples were crashed 1:20 (w/v) in extraction buffer using a motor and pestle. DASELISA plates were prepared by adding $200 \mu \mathrm{l}$ coating antibody for each specific MLN causing virus into each well of microtitre pate (dilution 1:200 v/v of antibody: buffer) followed by 2 hours of incubation at $37^{\circ} \mathrm{C}$. Plates were washed three times in PBS-T (Phosphate Buffered SalineTween $20 \mathrm{pH} 7.4$ ). A total of $200 \mu \mathrm{l}$ of the test samples were added into each well in duplicates and incubated at $4^{\circ} \mathrm{C}$ overnight. Plates were washed three times and $200 \mu$ l enzyme conjugate diluted in ECL buffer 1:200 (v/v) added to each well. Plates were incubated at $37^{\circ} \mathrm{C}$ for 3 hours and washed three times. A total $200 \mu \mathrm{l}$ freshly prepared substrate (1 $\mathrm{mg} / \mathrm{ml}$ paranitrophenyl-phosphate in substrate buffer) was added to each well, incubated at $37^{\circ} \mathrm{C}$ for 60 minutes. In the microtitre plates positive and negative control tests of healthy maize plants were included. The positive controls were purchased from Agdia Inc. (Elkhart, IN, USA). Plates were then assessed visually for colour change and absorbance measured at $405 \mathrm{~nm}$ wavelength using a $\mathrm{BIO}-\mathrm{RAD} \otimes$ microtitre plate reader Model 680 (BIO-RAD Laboratories, 
Hercules, California, USA). All samples were assayed in duplicate and the results judged to be positive if the absorbance was greater than or equal to twice the average reading of the negative (healthy) controls.

\begin{abstract}
Molecular detection of Maize lethal necrosis causing viruses in weeds and cultivated crops: Total RNA was extracted from leaves of weeds and cultivated crops with Trizol Reagent (Bioneer, South Korea) according to the manufacturer's instructions and subsequently used for cDNA synthesis by RT-PCR using AccuPower Reverse Transcription Polymerase Chain Reaction (RT-PCR) PreMix kit (Bioneer Corporation, Korea) following manufacturer's instructions. MCMV and SCMV primers which flank the coat protein gene of each virus and amplify a fragment of approximately $550 \mathrm{bp}$ for MCMV and $900 \mathrm{bp}$ for SCMV were used for RT-PCR [3]. Electrophoresis was done on the RT-PCR product using 1.5\% agarose gels for 45 minutes at $120 \mathrm{~V}$ and current of $400 \mathrm{~mA}$ in TAE agarose gel. The amplified DNA fragments were visualized on a $1.5 \%$ agarose gels under UV light. A $100 \mathrm{bp}$ DNA Ladder (Bioneer®) was used as the standard.
\end{abstract}

\subsection{Data Collection and Analysis}

Data was collected on the frequency of occurrence of weeds and cultivated crop species in each sampled field. In order to determine disease incidence, data on disease incidence (\%) was expressed as being equal to the total number of infected plants as a percentage of the total number of plants tested [21]. The collected data were arranged using Excel to generate datasets. The datasets were then imported into SPSS. The survey data obtained were analyzed using Statistical Package for Social Sciences (SPSS, version 20.0. Armonk, New York: IBM Corporation). The frequency of occurrence of weeds and crops for each agroecological region and district were analyzed using cross tabulation in the Statistical Package for Social Sciences (SPSS, version 20.0. Armonk, New York: IBM Corporation). In addition to descriptive statistics, further analysis was required to establish whether there were significant association between occurrence of weed species and specific agroecological zones. Therefore associations between occurrence of weed species and specific agroecological zones were tested using Pearson's chi-square tests.

\section{RESULTS}

\subsection{Survey of Weed and Cultivated Crop Species Found Growing in Association with Maize in Major Maize Growing Agroecological Zones in Uganda}

A total of 16 species of weeds representing 8 families were found in the major maize growing agroecological zones in Uganda (Table 1). The family Poaceae had the highest (8) number of species followed by Asteraceae with two species. The other families namely Amaranthaceae, Commelinaceae, Cyperaceae, Euphorbiaceae and Oxalidaceae each had one species. A total of 17 cultivated crops species representing 9 families were found in the major maize growing agroecological zones of Uganda (Table 2). The family Fabaceae had the highest (5) number of species followed by Solanaceae with three species and Poaceae with three species. The other families namely Musaceae, Pedaliaceae, Euphorbiaceae, Convolvulaceae, Cucurbitaceae each had one species. Most of the crop species identified were annuals (15 species) while 2 were perennials (Table 2).

Digitaria abyssinica, Bidens pilosa and Commelina benghalensis were the most frequently found weed species across the five major maize growing agroecological zones in Uganda (Table 3). The frequency of occurrence of most weed species notably Striga hermonthica $\left(x^{2}=173.9, \mathrm{df}=4, \mathrm{P}>0.0001\right)$, Digitaria abyssinica $\left(x^{2}=35.4\right.$, df $\left.=4, \mathrm{P}>0.0001\right)$, Amaranthus spinosus $\left(x^{2}=86.3\right.$, df $=4, \mathrm{P}>$ $0.0001)$, Bidens pilosa $\left(x^{2}=49.4\right.$, df $=4, \mathrm{P}>$ $0.0001)$, Pennisetum purpureum $\left(x^{2}=28.5\right.$, df $=4, P>0.0001)$, Panicum maximum $\left(x^{2}=19.3\right.$, $\mathrm{df}=4, \mathrm{P}>0.001)$, Oxalis latifolia $\left(x^{2}=17.4, \mathrm{df}=4\right.$, $P>0.02)$, Commelina benghalensis $\left(x^{2}=29.1\right.$, df $=4, \mathrm{P}>0.001)$, Imperata cylindrica $\left(x^{2}=35.1, \mathrm{df}\right.$ $=4, \mathrm{P}>0.001)$, Cyperus rotundus $\left(x^{2}=13.3\right.$, df $=4, \mathrm{P}>0.01)$, Chloris gayana $\left(x^{2}=24.4, \mathrm{df}=4, \mathrm{P}\right.$ $>0.001)$, Galinsonga parviflora $\left(x^{2}=28.6\right.$, df $=4$, $P>0.001)$, Pennisetum clandestinum $\left(x^{2}=30.5\right.$, $\mathrm{df}=4, \mathrm{P}>0.001)$ and Eleusine indica $\left(x^{2}=11.7\right.$, df $=4, P>0.019$ ) in farms is significantly associated with a specific agroecological zone (Table 3). However the frequency of occurrence of Euphorbia heterohylla Linn species was not significantly $\left(x^{2}=4.246\right.$, df $\left.=4, \mathrm{P}>0.05\right)$ associated with specific agroecological zones. The highest frequency of occurrence of weed species was found in the Eastern Highland Agroecological Zone (432), followed by the Lake 
Table 1. Potential weed hosts of MLN viruses identified in 5 major maize agroecological zones in Uganda during surveys conducted from 2014 to 2015

\begin{tabular}{|c|c|c|c|c|}
\hline Family & Species & Common name & Life cycle & Type of weed \\
\hline Amaranthaceae & Amaranthus spinosus Linn & Thorny pigweed & Annual & Broad leaves \\
\hline \multirow[t]{2}{*}{ Asteraceae } & Bidens pilosa Linn. & Black jack & Annual & Broad leaves \\
\hline & Galinsonga parviflora Cav. & Gallant soldier & Annual & Broad leaves \\
\hline Commelinaceae & Commelina benghalensis (L.) & Wandering Jew & Annual & Broad leaves \\
\hline Euphorbiaceae & Euphorbia heterohylla Linn & Purge weed & Annual & Broad leaves \\
\hline Poaceae & Eleusine indica (L.) Gaert & Wild Finger Millet & Annual & Grasses \\
\hline Scrophulariaceae & Striga hermonthica & purple witch weed & Annual & Broad leaves \\
\hline Cyperaceae & Cyperus rotundus (L.) & Nutgrass & Perennial & Sedges \\
\hline Oxalidaceae & Oxalis latifolia Kunth & Broadleaf Woodsorrel & Perennial & Broad leaves \\
\hline \multirow[t]{6}{*}{ Poaceae } & Pennisetum purpureum & Elephant Grass & Perennial & Grasses \\
\hline & Panicum maximum Jacq. & Common Guinea Grass & Perennial & Grasses \\
\hline & Imperata cylindrica (L.) & Sword or Spear Grass & Perennial & Grasses \\
\hline & Cynodon dactylon (L.) Pers. & Common Star Grass & Perennial & Grasses \\
\hline & Pennisetum clandestinumChiov & Kikuyu Grass & Perennial & Grasses \\
\hline & Digitaria abyssinica (A.Rich) Stapf & African Couch Grass & Perennial & Grasses \\
\hline
\end{tabular}


Mudde et al.; ARRB, 32(3): 1-17, 2019; Article no.ARRB.49407

Table 2. Potential cultivated crops hosts of MLN viruses found growing as intercrops with maize in 5 major maize agroecological zones of Uganda over 3 seasons 2014-2015

\begin{tabular}{|c|c|c|c|c|c|}
\hline Family & Common name & Botanical name & Life cycle & Total & Proportion (\%) \\
\hline Amaryllidaceae & Onions & Allium cepa & Annual & 3 & 0.8 \\
\hline Convolvulaceae & Sweet potato & Ipomoea batatas & Annual & 13 & 3.4 \\
\hline Cucurbitaceae & Pumpkin & Cucurbita sp & Annual & 3 & 0.8 \\
\hline Euphorbiaceae & Cassava & Manihot esculenta & Perennial & 49 & 12.7 \\
\hline \multirow[t]{5}{*}{ Fabaceae } & Groundnuts & Arachis hypogaea & Annual & 37 & 9.6 \\
\hline & Soybeans & Glycine max & Annual & 13 & 3.4 \\
\hline & Common Beans & Phaseolus vulgaris & Annual & 189 & 49.1 \\
\hline & Mung bean & Vigna radiate & Annual & 1 & 0.3 \\
\hline & Cowpea & Vigna unguiculata & Annual & 5 & 1.3 \\
\hline Musaceae & Bananas & Musa sp & Perennial & 35 & 9.1 \\
\hline Pedaliaceae & Simsim & Sesamum indicum & Annual & 2 & 0.5 \\
\hline \multirow[t]{4}{*}{ Poaceae } & Finger millet & Eleusine coracana & Annual & 6 & 1.6 \\
\hline & Rice & Oryza sativa & Annual & 3 & 0.8 \\
\hline & Sorghum & Sorghum bicolor & Annual & 9 & 2.3 \\
\hline & Sugar cane & Saccharum officinarum & Annual & 13 & 3.4 \\
\hline \multirow[t]{3}{*}{ Solanaceae } & Bitter tomato & Solanum incanum & Annual & 1 & 0.3 \\
\hline & Potato & Solanum tuberosum & Annual & 1 & 0.3 \\
\hline & $\begin{array}{l}\text { Tomatoes } \\
\text { Total }\end{array}$ & Lycopersicum esculentum & Annual & $\begin{array}{l}2 \\
385\end{array}$ & $\begin{array}{l}0.5 \\
100.0\end{array}$ \\
\hline
\end{tabular}


Table 3. Distribution and frequency of occurrence of weed species in five major maize growing agroecological zones in Uganda during 3 seasons 2014, 2014 and 2015A

\begin{tabular}{|c|c|c|c|c|c|c|c|c|c|c|}
\hline Weed species & EAZ & EHZ & LAZ & SEZ & WHZ & Total $^{\mathrm{a}}$ & Proportion (\%) & $x^{2}$ & $\mathbf{P}$ & df \\
\hline Striga hermonthica & 24 & 7 & 6 & 68 & 4 & 109 & 9.3 & 173.9 & 0.0001 & 4 \\
\hline Digitaria abyssinica & 51 & 119 & 59 & 37 & 28 & 294 & 25.2 & 35.4 & 0.0001 & 4 \\
\hline Amaranthus spinosus & 5 & 26 & 3 & 5 & 30 & 69 & 5.9 & 86.3 & 0.0001 & 4 \\
\hline Bidens pilosa & 16 & 82 & 72 & 22 & 25 & 217 & 18.6 & 49.4 & 0.0001 & 4 \\
\hline Pennisetum purpureum & 2 & 8 & 19 & 1 & 4 & 34 & 2.9 & 28.5 & 0.0001 & 4 \\
\hline Panicum maximum & 0 & 3 & 8 & 0 & 0 & 11 & 0.9 & 19.3 & 0.001 & 4 \\
\hline Oxalis latifolia & 4 & 13 & 1 & 0 & 0 & 18 & 1.5 & 17.4 & 0.02 & 4 \\
\hline Commelina benghalensis & 20 & 61 & 10 & 16 & 15 & 122 & 10.5 & 29.1 & 0.001 & 4 \\
\hline Imperata cylindrica & 10 & 11 & 28 & 4 & 4 & 57 & 4.9 & 35.1 & 0.001 & 4 \\
\hline Cyperus rotundus & 4 & 6 & 0 & 0 & 0 & 10 & 0.9 & 13.3 & 0.01 & 4 \\
\hline Chloris gayana & 12 & 9 & 0 & 7 & 3 & 31 & 2.7 & 24.4 & 0.001 & 4 \\
\hline Galinsonga parviflora & 0 & 3 & 0 & 0 & 6 & 9 & 0.8 & 28.6 & 0.001 & 4 \\
\hline Euphorbia spp & 11 & 19 & 8 & 10 & 5 & 53 & 4.5 & 4.2 & 0.374 & 4 \\
\hline Pennisetum clandestinum & 2 & 27 & 1 & 4 & 11 & 45 & 3.9 & 30.5 & 0.001 & 4 \\
\hline Eleusine indica & 9 & 32 & 22 & 8 & 16 & 87 & 7.5 & 11.7 & 0.019 & 4 \\
\hline Total & 170 & 426 & 237 & 182 & 151 & 1166 & 100.0 & & & \\
\hline
\end{tabular}

EAZ= Eastern Agroecological zone, EHZ= Eastern highland Agroecological zone, LAZ= Lake Albert Crescent Zone, SEZ= South Eastern Agroecological zone, WHZ= Western Highland Agroecological zone. ${ }^{a}$ Total number of samples of specific weed species identified and tested 
Albert Crescent Zone (237), South Eastern (182), Eastern Agroecological Zone (170) and Western Highland agroecological zone (158). Among the observed weeds, 10 weed species occurred in all the agroecological zones and they included Pennisetum purpureum, Pennisetum clandestinum, Euphorbia spp, Imperata cylindrica, Amaranthus spinosus, Eleusine indica, Striga hermonthica, Commelina benghalensis, Bidens pilosa and Digitaria abyssinica (Table 3 )

As regards cultivated crops, Beans (Phaseolus vulgaris), Cassava (Manihot esculenta), Groundnuts (Arachis hypogaea), Bananas (Musa $s p)$,Soybeans(Glycine max) and Sweet potato (Ipomoea batatas) were the most frequently found cultivated crop species grown with maize over the three seasons surveys across the five major maize agroecological zones (Table 4). They were used as 'indicators' in subsequent screen house studies to determine the MLN virus host range of cultivated crops grown with maize. The frequency of occurrence of most cultivated crop species notably Phaseolus vulgaris, Manihot esculenta, Arachis hypogaea, Musa sp, Sorghum bicolor, Eleusine coracana, Vigna unguiculata, Oryza sativa, Cucurbita sp, Sesamum indicum and Saccharum officinarum were significantly associated with specific agroecological zones. However the occurrence of Allium cepa, Solanum tuberosum, Vigna radiate, Solanum incanum, Lycopersicum esculentum, Glycine max and Ipomoea batatas were not significantly associated with specific agroecological zones (Table 4). The highest number of crop species (136) was found in the Eastern Highland Agroecological Zone, followed by South Eastern Agroecological Zone (74), Lake Albert Crescent Zone (70), Eastern Agroecological Zone (51) and Western Highland Agroecological Zone (40).

\subsection{Occurrence of MLN Causing Viruses in Alternative Host Weeds Found in Maize during Surveys in Major Agroecological Zones in 2014A, 2014B and 2015A}

The entire potential alternate host weed collected from 16 districts in five major maize agroecological zones of Uganda were identified as 16 different species (Table 5). These weeds belonged to eight families namely: Scrophulariaceae, Poaceae, Amaranthaceae, Asteraceae, Oxalidaceae, Commelinaceae,
Cyperaceae and Euphorbiaceae. Of these 16 species, some showed symptoms suggesting viral infection (chlorotic mosaic), whereas others showed no symptoms. All the weed species collected were tested for MCMV and SCMV by DAS-ELISA. Of these weeds, five tested positive for MCMV. These included Digitaria abyssinica, Amaranthus spinosus and Pennisetum purpureum, Cyperus rotundus and Commelina benghalensis (Tables 5). The ELISA test indicated that, in 2014A and 2014B, only Pennisetum purpureum from Bulambuli district in Eastern Highland Agroecological zone tested positive for MCMV with an overall relatively high incidence of $63 \%$ or 5 infected plants samples out of 8 samples over the three seasons (Table 5). A greater number of naturally infected weeds species were identified in 2015A compared to the two previous seasons. Three new hosts species with a relatively lower incidence were identified in Digitaria abyssinica (2\%), Amaranthus spinosus (8\%) and Commelina benghalensis (2\%) from Bulambuli district in Eastern Highland agroecological zone, tested positive for MCMV. Two additional weed hosts species with moderately high incidence notably Eleusine indica $(22 \%)$ and Cyperus rotundus $(33 \%)$ from Tororo district in Eastern Agroecological zone also tested positive for MCMV in 2015A. None of the grasses tested positive for SCMV during all the three surveys conducted (Table 5). Digitaria abyssinica, Commelina benghalensis, Amaranthus spinosus and Pennisetum purpureum expressed virus symptoms, including chlorosis of leaves typical of MCMV (Fig. 2).

\subsection{Occurrence of MLN Causing Viruses in Cultivated Crops Found in Maize Agroecosystems during Surveys of Major Agroecological Zones in 2014A, 2014B and 2015A}

Of these crops, the following tested positive for MCMV; beans (Phaseolus vulgaris) from MLN infected field in Bulambuli district (Eastern Highland Agroecological zone), cassava (Manihot esculenta) from MLN infected field in Bulambuli district (Eastern Highland Agroecological zone) and simsim (Sesamum indicum) from MLN infected field in Tororo district found in Eastern Agroecological zone (Table 6). Of these crops, only Sorghum bicolor (29\%) from MLN infected field in Tororo district (Eastern Agroecological zone) and sweet potato from Nabongo subcounty, Bulambuli district 
Mudde et al.; ARRB, 32(3): 1-17, 2019; Article no.ARRB.49407

Table 4. Distribution and frequency of occurrence of cultivated crops gown as intercrops with maize in five major maize growing agroecological zones in Uganda during 3 consecutive seasons

\begin{tabular}{|c|c|c|c|c|c|c|c|c|c|c|}
\hline Species & EAZ & EHZ & LAZ & SEZ & WHZ & ${ }^{\mathrm{a}}$ Total number & Proportion (\%) & $x^{2}$ & $\mathbf{P}$ & df \\
\hline Phaseolus vulgaris & 21 & 90 & 19 & 44 & 15 & 189 & 49.2 & 37.68 & 0.001 & 4 \\
\hline Manihot esculenta & 6 & 10 & 20 & 11 & 2 & 49 & 12.8 & 14.91 & 0.005 & 4 \\
\hline Arachis hypogaea & 1 & 24 & 3 & 3 & 6 & 37 & 9.6 & 21.54 & 0.001 & 4 \\
\hline Musa sp & 0 & 6 & 17 & 1 & 11 & 35 & 9.1 & 38.00 & 0.001 & 4 \\
\hline Glycine max & 4 & 1 & 2 & 5 & 1 & 13 & 3.4 & 8.49 & 0.075 & 4 \\
\hline Ipomoea batatas & 2 & 2 & 4 & 3 & 1 & 12 & 3.1 & 2.24 & 0.692 & 4 \\
\hline Sorghum bicolor & 7 & 0 & 0 & 2 & 0 & 9 & 2.3 & 36.10 & 0.001 & 4 \\
\hline Eleusine coracana & 4 & 0 & 0 & 2 & 0 & 6 & 1.6 & 18.21 & 0.001 & 4 \\
\hline Vigna unguiculata & 4 & 0 & 1 & 0 & 0 & 5 & 1.3 & 21.03 & 0.001 & 4 \\
\hline Oryza sativa & 0 & 0 & 0 & 1 & 2 & 3 & 0.8 & 9.90 & 0.042 & 4 \\
\hline Cucurbita sp & 0 & 0 & 3 & 0 & 0 & 3 & 0.8 & 11.08 & 0.026 & 4 \\
\hline Allium cepa & 0 & 3 & 0 & 0 & 0 & 3 & 0.8 & 6.00 & 0.199 & 4 \\
\hline Lycopersicum esculentum & 0 & 0 & 1 & 0 & 1 & 2 & 0.5 & 4.56 & 0.336 & 4 \\
\hline Sesamum indicum & 2 & 0 & 0 & 0 & 0 & 2 & 0.5 & 13.62 & 0.009 & 4 \\
\hline Solanum tuberosum & 0 & 0 & 0 & 0 & 1 & 1 & 0.3 & 7.43 & 0.115 & 4 \\
\hline Vigna radiata & 0 & 0 & 0 & 1 & 0 & 1 & 0.3 & 3.90 & 0.420 & 4 \\
\hline Solanum incanum & 0 & 0 & 0 & 1 & 0 & 1 & 0.3 & 3.90 & 0.420 & 4 \\
\hline Saccharum officinarum & 0 & 6 & 0 & 0 & 7 & 13 & 3.4 & 28.02 & 0.0001 & 4 \\
\hline Total & 51 & 142 & 70 & 74 & 47 & 384 & 100.0 & & & \\
\hline
\end{tabular}

EAZ= Eastern Agroecological zone, EHZ= Eastern highland Agroecological zone, LAZ= Lake Albert Crescent Zone, SEZ= South Eastern Agroecological zone, WHZ= Western Highland Agroecological zone. ${ }^{a}$ Total number of samples of specific cultivated crop species identified and tested 
Table 5. Occurrence of Maize chlorotic mottle virus (MCMV) and Sugarcane mosaic virus (SCMV) in weed species collected from five agroecological zones in Uganda during first season 2014, second season 2014 and first season 2015

\begin{tabular}{|c|c|c|c|c|c|c|c|c|c|c|c|}
\hline & \multirow{2}{*}{$\begin{array}{l}{ }^{\mathrm{a}} \text { Total no. } \\
\text { of plants }\end{array}$} & \multicolumn{2}{|c|}{$\mathrm{EAZ}^{\mathrm{b}}$} & \multicolumn{2}{|c|}{$\mathrm{EHZ}^{\mathrm{b}}$} & \multicolumn{2}{|c|}{ LAZ $^{\mathbf{b}}$} & \multicolumn{2}{|c|}{ SEZ $^{\mathrm{b}}$} & \multicolumn{2}{|c|}{$\mathbf{W H Z}^{\mathbf{b}}$} \\
\hline & & $\begin{array}{l}\text { \% MCMV } \\
\text { infected }^{c}\end{array}$ & $\begin{array}{l}\text { \% SCMV } \\
\text { infected }^{c}\end{array}$ & $\begin{array}{l}\% \text { MCMV } \\
\text { infected }^{c}\end{array}$ & $\begin{array}{l}\text { \% SCMV } \\
\text { infected }^{c}\end{array}$ & $\begin{array}{l}\% \text { MCMV } \\
\text { infected }^{c}\end{array}$ & $\begin{array}{l}\text { \% SCMV } \\
\text { infected }^{c}\end{array}$ & $\begin{array}{l}\text { \%MCMV } \\
\text { infected }^{c}\end{array}$ & $\begin{array}{l}\text { \% SCMV } \\
\text { infected }^{c}\end{array}$ & $\begin{array}{l}\% \text { MCMV } \\
\text { infected }^{c}\end{array}$ & $\begin{array}{l}\text { \%SCMV } \\
\text { infected }^{c}\end{array}$ \\
\hline Striga hermonthica & 109 & $0 / 24(0)$ & $0 / 24(0)$ & $0 / 7(0)$ & $0 / 7(0)$ & $0 / 6(0)$ & $0 / 6(0)$ & $0 / 68(0)$ & $0 / 68(0)$ & $0 / 4(0)$ & $0 / 4(0)$ \\
\hline Digitaria abyssinica & 294 & $1 / 51(2)$ & $0 / 51(0)$ & $2 / 119(2)$ & $0 / 119(0)$ & $0 / 59(0)$ & $0 / 59(0)$ & $0 / 37(0)$ & 0/37 (0) & $0 / 28(0)$ & $0 / 28(0)$ \\
\hline Amaranthus spinosus & 69 & $0 / 5(0)$ & $0 / 5(0)$ & $2 / 26(8)$ & $0 / 26(0)$ & $0 / 3(0)$ & $0 / 3(0)$ & $0 / 5(0)$ & $0 / 5(0)$ & $0 / 30(0)$ & $0 / 30(0)$ \\
\hline Bidens pilosa & 217 & $0 / 16(0)$ & $0 / 16(0)$ & $0 / 85(0)$ & $0 / 82(0)$ & $0 / 72(0)$ & $0 / 72(0)$ & $0 / 22(0)$ & $0 / 22(0)$ & $0 / 25(0)$ & $0 / 25(0)$ \\
\hline Pennisetum purpureum & 34 & $0 / 2(0)$ & $0 / 2(0)$ & $5 / 8(63)$ & $0 / 8(0)$ & $0 / 19(0)$ & $0 / 19(0)$ & $0 / 1(0)$ & $0 / 1(0)$ & $0 / 4(0)$ & $0 / 4(0)$ \\
\hline Panicum maximum & 11 & $0 / 0(0)$ & $0 / 0(0)$ & $0 / 3(0)$ & $0 / 3(0)$ & $0 / 8(0)$ & $0 / 8(0)$ & $0 / 0(0)$ & $0 / 0(0)$ & $0 / 0(0)$ & $0 / 0(0)$ \\
\hline Oxalis Iatifolia & 18 & $0 / 4(0)$ & $0 / 4(0)$ & $0 / 0(0)$ & $0 / 13(0)$ & $0 / 1(0)$ & $0 / 1(0)$ & $0 / 0(0)$ & $0 / 0(0)$ & $0 / 0(0)$ & $0 / 0(0)$ \\
\hline Commelina benghalensis & 122 & $0 / 20(0)$ & $0 / 20(0)$ & $1 / 61(2)$ & $0 / 61(0)$ & $0 / 10(0)$ & $0 / 10(0)$ & $0 / 16(0)$ & $0 / 16(0)$ & $0 / 15(0)$ & $0 / 15(0)$ \\
\hline Imperata cylindrical & 57 & $0 / 10(0)$ & $0 / 10(0)$ & $0 / 11(0)$ & $0 / 11(0)$ & $0 / 28(0)$ & $0 / 28(0)$ & $0 / 4(0)$ & $0 / 4(0)$ & $0 / 4(0)$ & $0 / 4(0)$ \\
\hline Cyperus rotundus & 10 & $0 / 4(0)$ & $0 / 5(0)$ & $2 / 6(33)$ & $0 / 6(0)$ & $0 / 0(0)$ & $0 / 0(0)$ & $0 / 0(0)$ & $0 / 0(0)$ & $0 / 0(0)$ & $0 / 0(0)$ \\
\hline Cynodon dactylon & 31 & $0 / 12(0)$ & $0 / 12(0)$ & $0 / 9(0)$ & $0 / 9(0)$ & $0 / 0(0)$ & $0 / 0(0)$ & $0 / 7(0)$ & $0 / 7(0)$ & $0 / 3(0)$ & $0 / 3(0)$ \\
\hline Galinsonga parviflora & 9 & $0 / 0(0)$ & $0 / 0(0)$ & $0 / 3(0)$ & $0 / 3(0)$ & $0 / 0(0)$ & $0 / 0(0)$ & $0 / 0(0)$ & $0 / 0(0)$ & $0 / 6(0)$ & $0 / 6(0)$ \\
\hline Euphorbia heterohylla & 53 & $0 / 11(0)$ & $0 / 11(0)$ & $0 / 19(0)$ & $0 / 19(0)$ & $0 / 8(0)$ & $0 / 0(0)$ & $0 / 10(0)$ & $0 / 10(0)$ & $0 / 5(0)$ & $0 / 5(0)$ \\
\hline Pennisetum clandestinum & 45 & $0 / 2(0)$ & $0 / 2(0)$ & $0 / 27(0)$ & $0 / 27(0)$ & $0 / 1(0)$ & $0 / 1(0)$ & $0 / 4(0)$ & $0 / 4(0)$ & $0 / 11(0)$ & $0 / 11(0)$ \\
\hline Eleusine indica & 87 & $2 / 9(22)$ & $0 / 9(0)$ & $0 / 32(0)$ & $0 / 32(0)$ & $0 / 22(0)$ & $0 / 22(0)$ & $0 / 8(0)$ & $0 / 8(0)$ & $0 / 16(0)$ & $0 / 16(0)$ \\
\hline
\end{tabular}

South Eastern Agroecological zone, WHZ= Western Highland Agroecological zone. ${ }^{c}$ Numbers in parenthesis represent the percentage proportion of ELISA positive samples for MLN virus where MCMV= Maize chlorotic mottle virus and SCMV = Sugarcane mosaic virus 
Table 6. Occurrence of Maize chlorotic mottle virus (MCMV) and Sugarcane mosaic virus (SCMV) in cultivated crop species grown as intercrops with maize collected from five agroecological zones of Uganda during first and second season 2014 and first season 2015

\begin{tabular}{|c|c|c|c|c|c|c|c|c|c|c|c|}
\hline \multirow[t]{2}{*}{ Crop species } & \multirow{2}{*}{$\begin{array}{l}{ }^{\mathrm{a}} \text { Total no. } \\
\text { of samples }\end{array}$} & \multicolumn{2}{|c|}{${ }^{\mathrm{D}} \mathrm{EAZ}$} & \multicolumn{2}{|c|}{${ }^{\mathrm{D}} \mathrm{EHZ}$} & \multicolumn{2}{|c|}{${ }^{\mathrm{D}} \mathrm{LAZ}$} & \multicolumn{2}{|c|}{${ }^{\mathrm{D}} \mathrm{SEZ}$} & \multicolumn{2}{|c|}{${ }^{\mathrm{b}} \mathrm{WHZ}$} \\
\hline & & $\begin{array}{l}\text { \% MCMV } \\
\text { infected }^{c}\end{array}$ & $\begin{array}{l}\text { \% SCMV } \\
\text { infected }^{c}\end{array}$ & $\begin{array}{l}\text { \% MCMV } \\
\text { infected }^{c}\end{array}$ & $\begin{array}{l}\text { \% SCMV } \\
\text { infected }^{c}\end{array}$ & $\begin{array}{l}\text { \% MCMV } \\
\text { infected }^{c}\end{array}$ & $\begin{array}{l}\text { \% SCMV } \\
\text { infected }^{c}\end{array}$ & $\begin{array}{l}\text { \%MCMV } \\
\text { infected }^{c}\end{array}$ & $\begin{array}{l}\text { \% SCMV } \\
\text { infected }^{c}\end{array}$ & $\begin{array}{l}\text { \% MCMV } \\
\text { infected }^{c}\end{array}$ & $\begin{array}{l}\text { \% SCMV } \\
\text { infected }^{c}\end{array}$ \\
\hline Banana & 35 & $0 / 0(0)$ & $0 / 0(0)$ & $0 / 6(0)$ & $0 / 6(0)$ & $0 / 17(0)$ & $0 / 17(0)$ & $0 / 1(0)$ & $0 / 1(0)$ & $0 / 11(0)$ & $0 / 11(0)$ \\
\hline Bean & 189 & $0 / 21(0)$ & $0 / 21(0)$ & $2 / 90(2)$ & $0 / 90(0)$ & 0/19 (0) & $0 / 19(0)$ & $0 / 43(0)$ & $0 / 43(0)$ & $0 / 15(0)$ & $0 / 15(0)$ \\
\hline Cassava & 49 & $0 / 6(0)$ & $0 / 6(0)$ & $2 / 10(20)$ & $0 / 10(0)$ & $0 / 20(0)$ & $0 / 20(0)$ & $0 / 11(0)$ & $0 / 11(0)$ & $0 / 2(0)$ & $0 / 2(0)$ \\
\hline Cowpea & 5 & $0 / 4(0)$ & $0 / 4(0)$ & $0 / 0(0)$ & $0 / 0(0)$ & $0 / 1(0)$ & $0 / 1(0)$ & $0 / 0(0)$ & $0 / 0(0)$ & $0 / 0(0)$ & $0 / 0(0)$ \\
\hline Groundnut & 37 & $0 / 0(0)$ & $0 / 0(0)$ & $0 / 24(0)$ & $0 / 24(0)$ & $0 / 3(0)$ & $0 / 3(0)$ & $0 / 3(0)$ & $0 / 3(0)$ & $0 / 6(0)$ & $0 / 6(0)$ \\
\hline Irish potato & 1 & $0 / 0(0)$ & $0 / 0(0)$ & $0 / 0(0)$ & $0 / 0(0)$ & $0 / 0(0)$ & $0 / 0(0)$ & $0 / 0(0)$ & $0 / 0(0)$ & 0/1 (0) & 0/1 (0) \\
\hline Millet & 6 & $0 / 4(0)$ & $0 / 4(0)$ & $0 / 0(0)$ & $0 / 0(0)$ & $0 / 0(0)$ & $0 / 0(0)$ & $0 / 2(0)$ & $0 / 2(0)$ & $0 / 0(0)$ & $0 / 0(0)$ \\
\hline Onion & 2 & $0 / 0(0)$ & 0/0 (0) & $0 / 2(0)$ & $0 / 2(0)$ & $0 / 0(0)$ & $0 / 0(0)$ & $0 / 0(0)$ & 0/0 (0) & 0/0 (0) & $0 / 0(0)$ \\
\hline Pumpkin & 2 & $0 / 0(0)$ & $0 / 0(0)$ & $0 / 0(0)$ & $0 / 0(0)$ & $0 / 2(0)$ & $0 / 2(0)$ & $0 / 0(0)$ & $0 / 0(0)$ & $0 / 0(0)$ & $0 / 0(0)$ \\
\hline Rice & 3 & $0 / 0(0)$ & $0 / 0(0)$ & $0 / 0(0)$ & $0 / 0(0)$ & 0/0 (0) & 0/0 (0) & 0/1 (0) & 0/1 (0) & $0 / 2(0)$ & $0 / 2(0)$ \\
\hline Simsim & 2 & $2 / 2(100)$ & $0 / 2(0)$ & $0 / 0(0)$ & $0 / 0(0)$ & $0 / 0(0)$ & $0 / 0(0)$ & $0 / 0(0)$ & $0 / 0(0)$ & $0 / 0(0)$ & $0 / 0(0)$ \\
\hline Sorghum & 9 & $0 / 7(0)$ & $2 / 7$ (29) & $0 / 0(0)$ & $0 / 0(0)$ & $0 / 0(0)$ & $0 / 0(0)$ & $0 / 2(0)$ & $0 / 2(0)$ & $0 / 0(0)$ & $0 / 0(0)$ \\
\hline Soybean & 13 & $0 / 4(0)$ & $0 / 4(0)$ & $0 / 1(0)$ & $0 / 1(0)$ & 0/2 (0) & $0 / 2(0)$ & $0 / 5(0)$ & $0 / 5(0)$ & 0/1 (0) & 0/1 (0) \\
\hline Sugarcane & 13 & $0 / 0(0)$ & $0 / 0(0)$ & $0 / 6(0)$ & $0 / 6(0)$ & $0 / 0(0)$ & $0 / 0(0)$ & $0 / 0(0)$ & $0 / 0(0)$ & $0 / 7(0)$ & $0 / 7(0)$ \\
\hline Sweet potato & 12 & $1 / 2(50)$ & $0 / 2(0)$ & $0 / 2(0)$ & $1 / 2(50)$ & $0 / 4(0)$ & $0 / 4(0)$ & 0/3 (0) & $0 / 3(0)$ & $0 / 1(0)$ & $0 / 1(0)$ \\
\hline Tomato & 2 & $0 / 0(0)$ & $0 / 0(0)$ & $0 / 0(0)$ & $0 / 0(0)$ & 0/1 (0) & $0 / 1(0)$ & $0 / 0(0)$ & $0 / 0(0)$ & $0 / 1(0)$ & 0/1 (0) \\
\hline Yam & 1 & $0 / 0(0)$ & $0 / 0(0)$ & $0 / 0(0)$ & $0 / 0(0)$ & $0 / 0(0)$ & $0 / 0(0)$ & $0 / 0(0)$ & $0 / 0(0)$ & $0 / 1(0)$ & $0 / 1(0)$ \\
\hline
\end{tabular}

${ }^{a}$ Total number of samples of specific crop identified and tested: ${ }^{b}$ Agroecological zones where; EAZ= Eastern Agroecological zone, EHZ= Eastern highland Agroecological zone, LAZ= Lake Albert Crescent Zone, SEZ= South Eastern Agroecological zone, WHZ= Western Highland Agroecological zone. ${ }^{c}$ Numbers in parenthesis represent the percentage proportion of ELISA positive samples for MLN virus where MCMV= Maize chlorotic mottle virus and SCMV= Sugarcane mosaic virus 
(Eastern highland agroecological zone) tested positive for SCMV using DAS ELISA. The ELISA test indicated that, in 2014A, none of the cultivated crops tested positive for MCMV. In 2014B, two samples of simsim tested positive for MCMV from Molo Sub County, Tororo district in Eastern Agroecological zone. In 2015A, two samples of beans from Simu subcounty, Bulambuli district in Eastern highland agroecological zone tested positive for MCMV. In addition, two cassava (Manihot esculenta) samples collected from the same location in Eastern highland agroecological zone tested positive for MCMV. In 2015A, two sorghum (Sorghum bicolor) samples collected from Molo subcounty in Tororo district (Eastern highland agroecological zone) and one sample of sweet potato (Ipomoea batatas) from Nabongo subcounty, Bulambuli district (Eastern Highland Agroecological Zone) tested positive for SCMV. Like in the weed species, a greater number of naturally infected cultivated crop species were identified in 2015A compared to the two previous seasons. The naturally infected cultivated crops were predominantly in the Eastern Agroecological zone and Eastern highland agroecological zones.

\subsection{Molecular Detection of MLN Causing Viruses from Collected Weeds}

Weed samples that tested positive for MCMV using DAS- ELISA were confirmed positive using PCR based on presence of bands as shown in representative gels for MCMV (Fig. 3). The band size for MCMV fragment was $550 \mathrm{bp}$. Amplicons of the expected size of $550 \mathrm{bp}$ were amplified from RT-PCR product of the following positively tested weed species notably Pennisetum purpureum, Digitaria abyssinica, Cyperus rotundus, Commelina benghalensis, Amaranthus spinosus, Eleusine indica. SCMV was not detected using RT-PCR in either weeds or cultivated crops hence results are not presented.

\section{DISCUSSION}

This study represents the first survey of potential and known alternative hosts of viruses causing MLN from a large geographic area covering five major maize agroecological zones of Uganda. As regards occurrence of potential weed hosts in major maize growing agroecological zones, this study showed occurrence of a wide range of weeds in the five major maize growing agroecological zones of Uganda. Digitaria abyssinica, Bidens pilosa and Commelina benghalensis were the most commonly occurring weed species in the major maize growing agroecological zones in Uganda. Similarly earlier studies have reported that Digitaria $s p p, B$. pilosa, C. benghalensis, I. cylindrica, and $P$. maximum, were the major weeds in Uganda [22]. The weeds represented eight plant families. Of these families, Poaceae had the highest number of species recorded. Most of the weed species that had previously been identified as hosts of Maize chlorotic mottle virus were also in the family Poaceae which is consistent with observations that that this family contains large numbers of plants susceptible to MLN causing viruses $[3,7]$. The study also identified a number of known alternate hosts of SCMV as reported by [13] notably Chloris gayana, Cynodon dactylon, Oryza sativa, Panicum maximum, Saccharum officinarum, Sorghum bicolor and Zea mays found present in the major maize agroecological zones in Uganda. Most of these

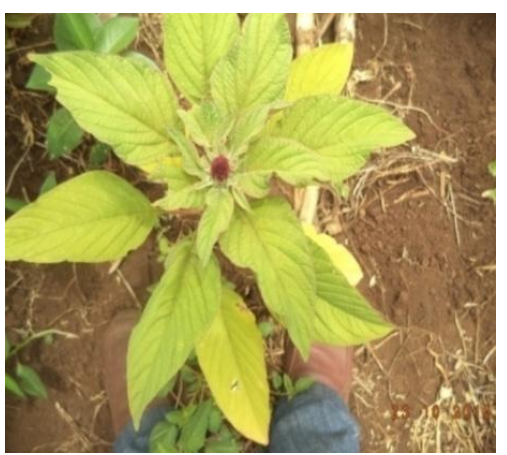

Amaranthus spinosus

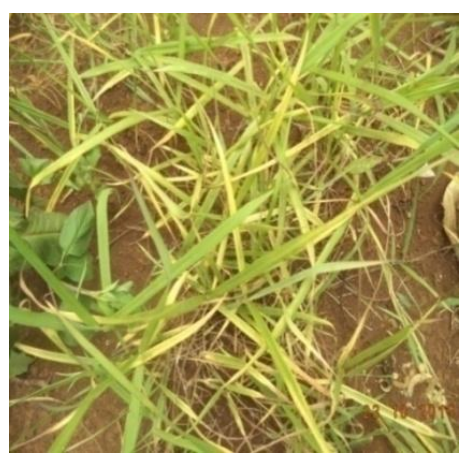

Digitaria abyssinica

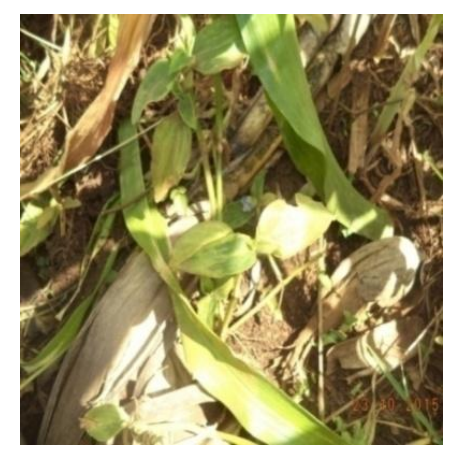

Commelina benghalensis

Fig. 2. Examples of MCMV virus-like chlorotic symptoms observed in alternative weed hosts found in MLN infected fields in Bulambuli district eastern Uganda 


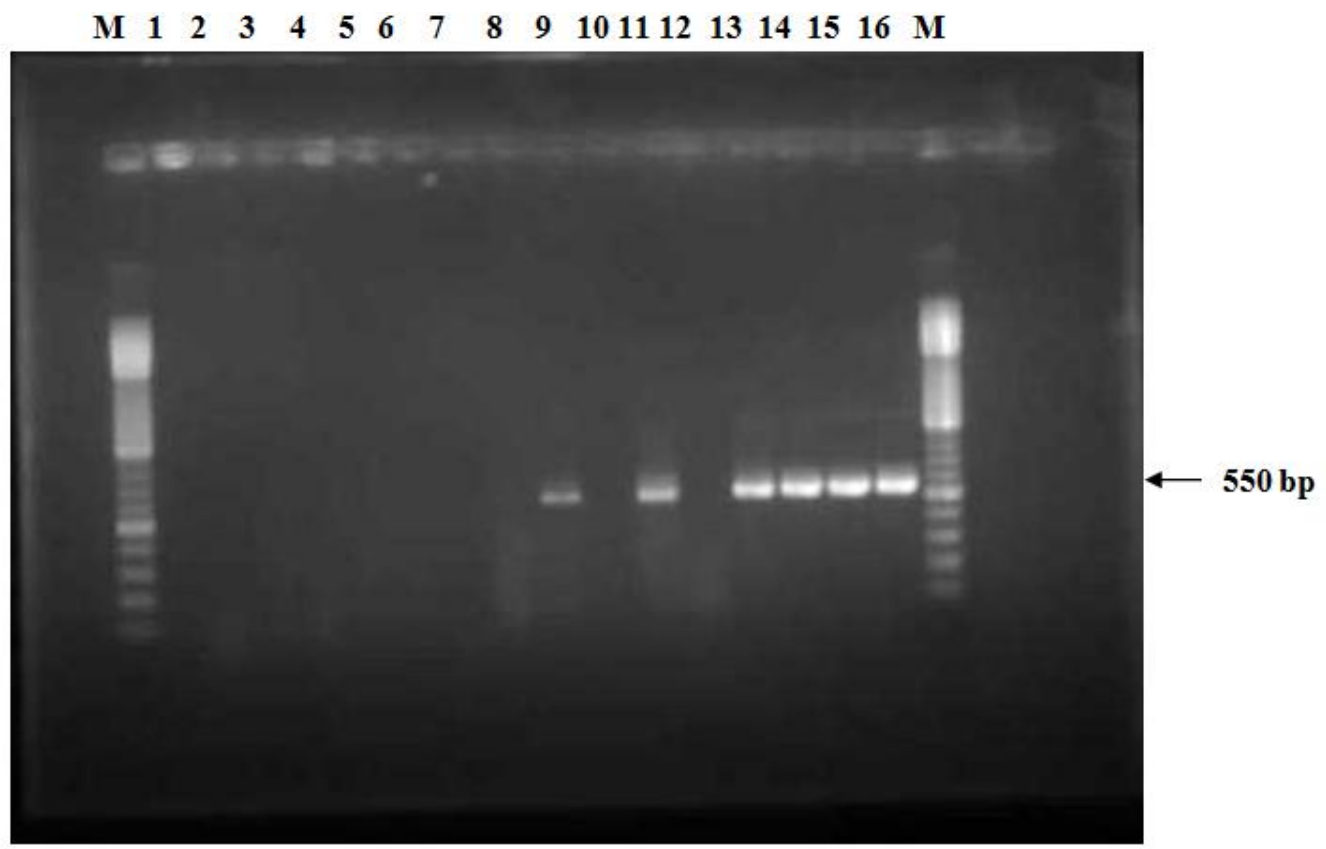

Fig. 3. RT-PCR products of MCMV in weed samples collected from major maize agroecological zones in Uganda. Lane $M$ represents $100 \mathrm{bp}$ DNA ladder (Bioneer). Lane 1=

Striga hermonthica, 2= Bidens pilosa, 3= Oxalis latifolia, 4= Galinsonga parviflora, 5= Saccharum officinarum, 6= Euphorbia spp, 7= Euphorbia heterohylla , 8= Chloris gayana, 9=Digitaria abyssinica, 10=Negative control (nuclease free water), 11= Amaranthus spinosus, 12= Panicum maximum, 13= Pennisetum purpureum, 14= Cyperus rotundus, 15= Commelina benghalensis, $16=$ Positive control (maize)

weed species are perennial in nature and can hence act as sources of inoculum. The several new virus weed-hosts identified suggests the availability of favorable hosts with the ability to harbor the MLN causing viruses and serve as sources of inoculum to its vectors. Perennial weed species can act as continuous endemic source of inoculum of virus and can be transferred to annual weeds where the virus propagates before being spread further to crops that are susceptible [23,24]. This implies that such weeds should not be overlooked when developing MLN management strategies.

Results of this study indicated that several weed species from the Poaceae family mainly collected from Eastern Highland and Eastern Agroecological Zones are susceptible to MLN causing viruses. A total of five weed species and three cultivated crops species were identified as natural alternative hosts of Maize chlorotic mottle virus a key driver of the MLN epidemic for the first time in Uganda. In the field survey conducted, Digitaria abyssinica, Amaranthus spinosus, Cyperus rotundus, Pennisetum purpureum and Commelina benghalensis were found to be naturally susceptible to MCMV. These results are in conformity with earlier reports only for Pennisetum purpureum which was reported to be a natural host for MCMV [16]. The results were not expected for the Commelinaceae and Amaranthaceae since MCMV had only been reported to be found in Poaceae family [7]. No plants in the families Commelinaceae and Amaranthaceae have hitherto been documented as hosts of MCMV. Furthermore, this appears to be the first observation of a large number of naturally MCMV-infected species in proximity to maize crops in the field, notably from the MLN hotspot districts of Bulambuli and Tororo. However there is no existence of MLN viruses in weeds found in the areas without disease pressure. These facts suggest that the continuously high incidence of this virus in these MLN hotspot areas may be partially associated with several alternative MCMV weed host sources in these maizeproducing agroecological zones. Up to the recent past when MCMV was identified in sorghum $[25,26]$, sugarcane [8], finger millet [9], Napier grass [16] and Kikuyu grass [16], the only naturally occurring host of MCMV was maize [7]. 
This is therefore the first report of MCMV in Digitaria abyssinica, Amaranthus spinosus and Commelina benghalensis.

As regards surveys of potential natural hosts of MLN viruses in cultivated crops, the following tested positive for MCMV; beans (Phaseolus vulgaris) and cassava (Manihot esculenta), from MLN infected field in Bulambuli district (Eastern Highland Agroecological zone) and Simsim from MLN infected field in Tororo district (Eastern Agroecological zone). Out of the studied crops, only sorghum from MLN infected field in Tororo district (Eastern Agroecological zone) and sweet potato from Nabongo subcounty, Bulambuli district (Eastern highland agroecological zone) tested positive for SCMV using DAS ELISA. The findings agree with earlier reports showing sorghum is a natural host of SCMV [27]. However, the cultivated plants did not show symptoms related to SCMV which suggests they could be resistant to infection. The results were not expected for those cultivated crops which are dicotyledonous plants from non graminae families. Prior to this, MCMV has only known to be found in the Poaceae family [7] Cassava, beans and groundnuts are dicotyledonous plants $[28,29]$. These results do not support previous findings that reported that dicotyledonous species were not mechanically infected with MCMV $[30,31]$. Specific isolate-host interactions could probably explain the contrasting results observed with some plant species about their host status for MLN causing viruses. However, these results are in conformity with previous studies that showed that some dicotyledonous plants can be natural and artificial hosts of MCMV [32]. Nonetheless the findings in this study suggest that these cultivated crops may carry the virus based on the virus titers that were comparable to the positive maize control. Prior to this study, no weeds and crop species were found to be naturally affected in the wild probably because they are not favorable hosts for vectors of MCMV. These ELISA based results were not confirmed in most of the weed species using PCR for SCMV but only for MCMV. These findings are in conformity to findings in Kenya that also reported low detection of SCMV using PCR despite positive results using ELISA $[1,16]$. This is probably due to the emergence of new strains of SCMV with sequences in capsid protein that are different from the sequences used to design the primers used. Indeed studies have confirmed that SCMV strains in the East African region are highly divergent [1].
This study could hence provide the first evidence of the potential role of cultivated crops as hosts of MLN causing viruses. There is need for further studies on these cultivated crops to investigate whether they share vectors of MCMV with maize and these vectors can transfer the virus from the maize to these crops and vice versa. No information is available concerning the occurrence of natural sources of MLN causing viruses in cultivated crops including beans (Phaseolus vulgaris), cassava (Manihot esculenta), groundnuts (Arachis hypogaea) bananas (Musa sp), soybeans (Glycine max) and sweet potato (Ipomoea batatas) and hence these findings provide the first report of the potential role these cultivated crops could play as reservoirs of MCMV potentially increasing the amount of virus innoculum within the field. The implication of these findings is that crops like sorghum and cassava commonly grown in these areas have some varieties that are late maturing and can hence provide a source of innoculum to the next season crop of maize. Furthermore, beans are commonly grown as intercrops with maize and hence could also potentially provide a source of inoculum of MLN causing virus when grown with maize. In addition to this, sweet potato is a late planted crop in most cropping systems of Uganda and hence could also provide a source of inoculum for the MLN viruses in the subsequent season crop. However, the importance of the cultivated crops as alternate hosts needs further studies to determine if vectors that can survive on maize can also survive on these alternative hosts.

\section{CONCLUSION}

The overall aim of this study was to identify alternative host weeds and crops species occurring in maize and their role in the spread of maize lethal necrosis-causing viruses in Uganda. It was hypothesized that potential alternative hosts of Maize Lethal Necrosis-causing viruses are present in major maize growing agroecological zones of Uganda and act as sources of inoculum to maize fields. The study also established the following natural weed hosts of MCMV, and they included; Digitaria abyssinica, Amaranthus spinosus, Cyperus rotundus, Pennisetum purpureum and Commelina benghalensis. The natural hosts for SCMV were only Sorghum and sweet potato. No natural hosts of SCMV were detected among the weeds. Based on these observations, these could be the most likely sources of MLN virus inoculum during the period when maize has 
been harvested and hence contributing to the spread of the MLN disease. The study has confirmed the existence of potential natural sources of MCMV innoculum in cultivated crops beans (Phaseolus vulgaris), cassava (Manihot esculenta) and Simsim (Sesamum indicum) obtained from MLN hotspot districts of Bulambuli and Tororo. Mechanical inoculation studies corroborated these findings in cassava (Manihot esculenta), groundnuts (Arachis hypogaea) and beans (Phaseolus vulgaris). Existence of alternative hosts may explain early infection of maize plants by MCMV and SCMV and the continued occurrence of the MLN disease in the hotspot districts of eastern Uganda. Therefore, this information serves as justification for regular weed management in maize fields, as an Integrated Pest Management (IPM) option for the sustainable control of MLN.

\section{ACKNOWLEDGEMENT}

The authors acknowledge National Agricultural Research Organization (NARO) through Agricultural Technology and Agribusiness Advisory Services Project for research funding. Special thanks to Mrs. Olivia Wannyana from Botany Department-Makerere University for technical support in plant species identification.

\section{COMPETING INTERESTS}

Authors have declared that no competing interests exist.

\section{REFERENCES}

1. Adams IP, Harju VA, Hodges T, Hany U, Skelton A, Rai S, Deka MK, Smith J, Fox A, Uzayisenga B, Ngaboyisonga C, Uwumukiza B, Rutikanga A, Rutherford M, Ricthis B, Phiri N, Boonham N. First Report of Maize Lethal Necrosis Disease in Rwanda. New Dis. Rep. 2014;29(22). Avilable:http://dx.doi.org/10.5197/j.20440588.2014.029.022

2. Kitenge K. Current status of maize lethal necrosis disease in Tanzania. Paper Presented during Regional Workshop on Maize Lethal Necrosis and Its Management, Nairobi Safari Club, Kenya, February 12-14th, 2013; 2012.

3. Wangai AW, Redinbaugh MG, Kinyua ZM, Miano DW, Leley PK, Kasina M, Mahuku G, Scheets K, Jeffers D. First report of Maize chlorotic mottle virus and maize lethal necrosis in Kenya. Plant Dis. 2012;96(10):1582-1583.
Available:https://doi.org/10.1094/PDIS-0612-0576-PDN

4. IPPC. New pest of maize: Maize lethal necrosis in Uganda. IPPC Official Pest Report, No. UGA-01/2, No.UGA-01/2. Rome, Italy: FAO; 2014.

Available:https://www.ippc.int/

5. Asea G. MLN in Uganda: A disease on the move. A Paper Presented during Workshop on Maize Lethal Necrosis and Its Management, February $12-14^{\text {th }} 2013$, Nairobi Safari Club; 2013.

6. Lukanda $M$, Owati $A$, Ogunsanya $P$, Valimunzigha $\mathrm{K}$, Katsongo $\mathrm{K}$, Ndemere $\mathrm{H}$, Kumar PL. First report of Maize chlorotic mottle virus infecting maize in the Democratic Republic of the Congo. Plant Dis. 2014;98(10):1448-1449.

Available:https://doi.org/10.1094/PDIS-0514-0484-PDN

7. Scheets K. Maize chlorotic mottle virus. In Viruses and Virus Diseases of Poaceae (Gramineae), H. Lapierre and P.A. Signoret, Eds. 2004;642-644.

8. Wang Q, Zhou XP, Wu JX. First report of Maize chlorotic mottle virus infecting sugarcane (Saccharum officinarum). Plant Dis. 2014;98(4):572-572.

Available:https://doi.org/10.1094/PDIS-0713-0727-PDN

9. Kusia ES, Subramanian S, Nyasani JO, Khamis F, Villinger J, Ateka EM, Pappu HR. First report of lethal necrosis disease associated with co-infection of finger millet with Maize chlorotic mottle virus and Sugarcane mosaic virus in Kenya. Plant Dis. 2015;99(6):899-900.

Available:http://dx.doi.org/10.1094/PDIS10-14-1048-PDN

10. Bockelman DL, Claflin LE, Uyemoto JK. Host range and seed-transmission studies of Maize chlorotic mottle virus in grasses and corn. Plant Dis. 1982;66(3):216-218.

DOI: 10.1094/PD-66-216

11. Nelson S, Brewbaker J, $\mathrm{Hu}$ J. Maize chlorotic mottle virus. Plant Dis. 2011;79:16.

12. Rao GP, Jain RK, Varma A. Occurrence of maize dwarf mosaic virus on maize and Sudan grass in India. Plant Dis. 1996;80(6):711.

13. Brunt A, Crabtree K, Gibbs A. Viruses of tropical plants. Wallingford, Oxon, UK: CAB International. 1990;707.

14. Toler RW. Maize dwarf mosaic, the most important virus disease of sorghum. Plant Dis. 1985;69(11):1011-1015. 
15. Kusia Elizabeth Siago. Characterization of Maize chlorotic mottle virus and sugarcane mosaic virus causing maize lethal necrosis disease and spatial distribution of their alternative hosts in Kenya. Msc. Thesis; 2014.

16. Mahuku G, Lockhart BE, Wanjala B, Jones MW, Kimunye JN, Stewart LR, Cassone BJ, Sevgan S, Nyasani JO, Kusia E, Kumar PL, Niblett CL, Kiggundu A, Asea G, Pappu HR, Wangai A, Prasanna BM, Redinbaugh M. Maize Lethal Necrosis (MLN), an emerging threat to maize-based food security in Sub-Saharan Africa. Phytopathology. 2015;105(7):956-965.

Available:https://doi.org/10.1094/PHYTO12-14-0367-FI

17. Wottman CS, Eledu CA. Uganda's agroecological zones. A guide to planners and policy makers. Centro Internationale due Agricultural Tropical CIAT, Kawanda, Uganda; 1999.

18. Phillips $S$, Namaganda $M$, Lye KA. Ugandan Grasses. Department of Botany, Makerere University. 2003;115.

19. Mudde B, Olubayo FMM, Miano DW, Asea G, Kilalo DC, Kiggundu A, Bomet DK, Adriko J. Distribution, incidence and severity of maize lethal necrosis disease in major maize growing agro-ecological zones of Uganda. J. Agric. Sci. 2018;10(6): 72.

20. Clark MF, Adams AN. Characteristics of the microplate method of enzyme-linked immunosorbent assay for the detection of plant viruses. J. Gen. Virol. 1977;34:475483.

DOI: 10.1099/0022-1317-34-3-475

21. Biswanath Das, Daniel Jeffers, George Mahuku, Yoseph Beyene, Dan Makumbi, Anne Wangai. Standardized screening protocols for MLN. A Paper Presented during a Regional Workshop on Maize Lethal Necrosis and Its Management Strategies, February $12-14^{\text {th }} 2013$, Nairobi Safari Club; 2013.
22. Terry PJ, Webb M. Weed problems in Uganda: Report of Visit 5-7 May. NRI International Document; 1992.

23. Persley DM, Thomas JE, Sharman M. Tospoviruses - an Australian perspective. Australas Plant Path. 2006;35:161-180. Available:https://doi.org/10.1071/AP0601 5

24. Wilson CR. Incidence of weed reservoirs and vectors of tomato spotted wilt tospovirus on southern Tasmanian lettuce farms. Plant Path. 1998;47:171-176.

Available:https://doi.org/10.1046/j.13653059.1998.00227.x

25. Huang J, Wen GS, Li MJ, Sun CC, Sun Y, Zhao MF, He YQ. First report of Maize chlorotic mottle virus naturally infecting sorghum and coix seed in China. Plant Dis. 2016;100(9):1955-1955.

Available:https://doi.org/10.1094/PDIS-0216-0251-PDN

26. Achon MA, Serrano L, Clemente-Orta G, Sossai S. First report of Maize chlorotic mottle virus on a Perennial Host, Sorghum halepense, and Maize in Spain. Plant Dis. 2017;101(2):393-393.

27. Louie R. Sugarcane mosaic virus in Kenya. Plant Dis. 1980;64:944.

28. Purseglove JW. Tropical crops. Monocotyledons, 1. Longman, London; 1972.

29. Pursglove JW. Tropical crops dicotyledons I. longamans. Green \& Co. Ltd. London \& Harlow; 1969.

30. Castillo J, Herbert TT. New virus disease affecting maize in Peru. Phytopathology. 1974;38(4):184-189.

31. Niblett CL, Claflin LE. Corn lethal necrosis - a new virus disease of corn in Kansas. Plant Dis. Rep. 1978;62:15-19.

32. Jiang $X Q$, Meinke LJ, Wright RJ, Wilkinson DR, Campbell JE. Maize chlorotic mottle virus in Hawaiian-grown maize: Vector relations, host range and associated viruses. Crop Prot. 1992;11(3):248-254. Available:https://doi.org/10.1016/02612194(92)90045-7

(c) 2019 Mudde et al.; This is an Open Access article distributed under the terms of the Creative Commons Attribution License (http://creativecommons.org/licenses/by/4.0), which permits unrestricted use, distribution, and reproduction in any medium, provided the original work is properly cited.

Peer-review history:

The peer review history for this paper can be accessed here: http://www. sdiarticle3.com/review-history/49407 